\title{
Abnormal medial prefrontal cortex functional connectivity and its association with clinical symptoms in chronic low back pain
}

\author{
Yiheng Tu, $\mathrm{PhD}^{1,2}$, Minyoung Jung, PhD ${ }^{1}$, Randy L. Gollub, MD, PhD ${ }^{1}$, Vitaly Napadow, \\ PhD $^{2}$, Jessica Gerber, PhD $^{2}$, Ana Ortiz, BA ${ }^{1}$, Courtney Lang, BA ${ }^{1}$, Ishtiaq Mawla, BA ${ }^{1}$, Wei \\ Shen, MD, PhD ${ }^{1}$, Suk-Tak Chan, PhD ${ }^{2}$, Ajay D. Wasan, MD ${ }^{3}$, Robert R. Edwards, PhD $^{4}$, Ted \\ J. Kaptchuk, BA ${ }^{4}$, Bruce Rosen, MD, PhD², and Jian Kong, MD, MS, MPH ${ }^{1,2}$ \\ ${ }^{1}$ Department of Psychiatry, Massachusetts General Hospital, Harvard Medical School, \\ Charlestown, MA, USA \\ ${ }^{2}$ Department of Radiology, Martinos Center for Biomedical Imaging, Massachusetts General \\ Hospital, Harvard Medical School, Charlestown, MA, USA \\ ${ }^{3}$ Department of Anesthesiology, Center for Pain Research, University of Pittsburgh, Pittsburgh, \\ PA, USA \\ ${ }^{4}$ Department of Anesthesiology, Perioperative and Pain Medicine, Brigham and Women's \\ Hospital, Harvard Medical School, Boston, MA, USA
}

\begin{abstract}
Accumulating evidence has shown that complicated brain systems are involved in the development and maintenance of chronic low back pain (cLBP), but its underlying mechanisms, particularly the association between brain functional changes and clinical outcomes, remain unclear. Here we used resting-state fMRI and multivariate pattern analysis (MVPA) to identify abnormal functional connectivity (FC) between the default mode, sensorimotor, salience and central executive brain networks in cLBP and tested whether abnormal FCs are related to pain and comorbid symptoms. Fifty cLBP patients and 44 matched healthy controls (HCs) underwent an fMRI scan, from which brain networks were identified by independent component analysis. MVPA, graph theory approaches, and correlation analyses were applied to find abnormal FCs that were associated with clinical symptoms. Findings were validated on a second cohort of $30 \mathrm{cLBP}$ patients and 30 matched HCs. Results showed the medial prefrontal cortex (mPFC) had abnormal FCs with brain regions within the DMN and with other brain networks in cLBP patients. These altered FCs were also correlated with pain duration, pain severity, and pain interference. Lastly, we found restingstate FC could discriminate cLBP patients from HCs with 91\% accuracy in the first cohort and $78 \%$ accuracy in the validation cohort. Our findings suggest the mPFC may be an important hub for linking the default mode network with the other three networks in cLBP patients. Elucidating the altered FCs and their association with clinical outcomes will enhance our understanding of the pathophysiology of cLBP and may facilitate the development of pain management approaches.
\end{abstract}

Corresponding Author: Jian Kong, Department of Psychiatry, Massachusetts General Hospital, Charlestown, MA 02129, jkong2@mgh.harvard.edu, Phone: +1 617-286-9879.

J.K. has a disclosure to report that is not relevant to this study (holding equity in a startup company and pending patents to develop new neuromodulation devices) but claims no conflict of interest. All other authors declare no conflict of interest. 


\section{Keywords}

chronic low back pain; multivariate pattern analysis; medial prefrontal cortex; clinical symptoms; classification

\section{Introduction}

Chronic low back pain (cLBP) is one of the most common reasons for physician visits in the USA [26] and the leading cause of disability globally [63]. Current treatments for cLBP are often unsatisfactory. Few new non-opioid and non-addictive pain medications have been developed in over five decades [41]. This is at least partly due to the limited understanding of the underlying mechanisms of development and maintenance of cLBP.

Neuroimaging research has demonstrated definitive involvement of the central nervous system in the development, maintenance, and experience of chronic pain [42]. The brain of chronic pain patients is continuously processing spontaneous background pain by integrating information between multiple brain regions related to sensory, cognitive and emotional functions [56]. Resting-state fMRI provides a useful framework for investigating the neural mechanisms associated with chronic pain by comparing brain activities and networks between patients and healthy controls (HCs). For example, the default mode network (DMN), thought to be involved in higher order functions and monitoring the internal environment for salient events, has consistently been found to be disrupted in cLBP $[4,9,40]$. The salience network (SN), which monitors changes in sensory input and coordinates brain activity to facilitate a behavioral response, has been shown to be abnormal in cLBP [12,27]. Also, abnormal connectivity within the central executive network (CEN), which is involved in cognitive control of behavior $[23,44]$, and the sensorimotor network (SMN), which processes sensory information [22,33], has been reported in cLBP and other chronic pain conditions.

Although previous studies have investigated abnormal resting-state networks in chronic pain conditions, several questions remain unanswered. For instance, many studies focused on only one network or used a predefined seed to study seed-to-voxel connectivity. However, chronic pain may be associated with alteration of multiple brain networks that comprise the pain connectome, including the ascending and descending modulation pathway (e.g., periaqueductal grey [PAG], thalamus), SN, CEN, and DMN [27,35,36]. The pain connectome emphasizes the integration of cognitive, affective and sensorimotor aspects of pain, but how these networks interact with each other remains poorly understood. Also, few studies have included an independent dataset to validate their findings.

In this study, resting-state functional connectivity ( $\mathrm{rsFC}$ ) within and across networks involving pain modulation and processing was used to test the hypotheses that cLBP patients have abnormal brain connectivity across multiple brain networks and abnormal rsFCs in the pain connectome are correlated with clinical symptoms of cLBP. Fifty cLBP patients and 44 matched HCs underwent an MRI scan. We selected four networks (DMN, SN, CEN, and SMN) using independent component analysis (ICA). A multivariate pattern analysis (MVPA) was applied to investigate and identify rsFCs that showed abnormities and were 
able to discriminate cLBP patients from HCs. Given the complex symptoms of cLBP, we related the abnormal rsFCs in cLBP with comprehensive clinical scores, including pain intensity and disorder-related characteristics (duration, pain severity), as well as comorbid physical, mental and social symptoms. In addition, we tested the validity of the findings using an independent dataset consisting of $30 \mathrm{cLBP}$ patients and $30 \mathrm{HCs}$.

\section{Materials and Methods}

\section{Participants}

This study included 50 patients diagnosed with cLBP with a duration of at least six months confirmed by a clinical evaluation and 44 matched HCs. We also included an independent dataset of $30 \mathrm{cLBP}$ patients and $30 \mathrm{HCs}$ for validation. All patients met the same inclusion criteria and were without other chronic pain comorbidities. Details of the inclusion criteria and medication usage for all participants can be found in Supplementary Materials. The Institutional Review Board (IRB) approved the studies, and all experiments were performed in accordance with the guidelines set forth by the IRB for ethics and protection of human subjects.

\section{Clinical assessments and medication}

The LBP severity assessment was used as the primary clinical measure in this study. It is a well-accepted assessment for chronic pain with adequate validity and excellent reliability $[52,69]$. It measures how bothersome a patient's LBP has been during the past week on a VAS scale $(0-10)$ from "not at all bothersome" to "extremely bothersome". Patients' pain intensity before the MRI scan was also measured with a 0 - 100 VAS scale from "no pain" to "worst pain imaginable".

We also used the Patient Reported Outcomes Measurement Information System (PROMIS-29) [16,17], which is a system of reliable, flexible and responsive assessment tools to assess physical, mental, and social health for cLBP patients. Seven items, including pain interference, physical health, social disability, sleep disturbance, fatigue, depression and anxiety, were tested in PROMIS and used to evaluate how chronic pain diminished patients' quality of life. In addition to PROMIS, patients' depression symptoms were assessed with the Beck Depression Inventory (BDI).

All included patients did not use prescription opioids greater than $60 \mathrm{mg}$ morphine equivalents per day or steroids for pain, and they were allowed to continue their existing medication. Six out of 50 patients took opioid analgesics in the first cohort. Four out of 30 patients took opioid analgesics in the second cohort. Details of medication information can be found in Supplementary Materials.

\section{MRI acquisition}

All functional MRI data were acquired using a 32-channel radio-frequency head coil in a 3T Siemens scanner at the Martinos Center for Biomedical Imaging. T2-weighted functional images encompassing the whole brain were acquired with the gradient-echo EPI sequence (echo time: $30 \mathrm{~ms}$, repetition time: $3,000 \mathrm{~ms}$, flip angle: $90^{\circ}$, slice thickness: $3 \mathrm{~mm}$, interslice 
gap: $0.88 \mathrm{~mm}, 44$ slices). During the 6-minute resting-state fMRI scan, subjects were asked to keep their eyes open and blink normally. High-resolution brain structural images were also acquired with a T1-weighted three-dimensional multi-echo magnetization-prepared rapid gradient-echo (MPRAGE) sequence (repetition time: 2,200 ms, echo time: $1.54 \mathrm{ms,}$ slice thickness $1 \mathrm{~mm}$, flip angle: $7^{\circ}$, and 176 sagittal slices covering the whole brain).

\section{fMRI preprocessing}

fMRI data were preprocessed using SPM12 (Wellcome Trust Centre for Neuroimaging, London, UK). The first five volumes were discarded for signal equilibration. Images were slice-timing corrected and realigned. The resulting images were normalized to the Montreal Neurological Institute (MNI) space [7] and spatially smoothed using a Gaussian kernel of 5 $\mathrm{mm}$ full width at half maximum (FWHM).

To minimize the effect of head motion on the estimation of functional connectivity, we followed a strategy suggested by a recent benchmark study [21] of combing 6 motion estimates and 2 physiological time series (mean signal in white matter and mean signal in cerebral spinal fluid [CSF]) as nuisance parameters and regressing them out from the wholebrain fMRI data for denoising. Since global signal regression (GSR) has drawn concerns regarding the potential for spurious negative connectivity [43,62], GSR was not applied in the present study. We also compared the maximal framewise displacement (FD) value between cLBP patients and HCs and did not find any significant difference [47]. Detailed results can be found in Supplementary Materials.

\section{Group ICA and functional connectivity construction}

The fMRI data were parcellated using spatial group independent component analysis (GICA) with the GIFT toolbox (Medical Image Analysis Lab, Albuquerque, New Mexico), and the whole-brain connectivity matrix was constructed based on the time courses (TCs) of intrinsic connectivity networks (ICNs). The procedure of performing GICA and identifying ICNs are shown in Figure 1.1. In the first step, principal component analysis (PCA) was applied to reduce the subject-specific data into 100 principle components, which preserved more than $99 \%$ of the variance. Next, reduced data of all subjects were concatenated across time and decomposed into 80 independent components (ICs) using the infomax algorithm. The infomax ICA algorithm was repeated 10 times in ICASSO (a software package incorporated in GIFT toolbox for performing ICA) [28], and the best run was selected to ensure estimation stability. After estimating the aggregate spatial maps, spatiotemporal regression back reconstruction was performed to obtain the subject specific spatial maps (SMs) and TCs.

After obtaining the SMs and TCs of all subjects, we calculated one-sample t-test maps for each SM across subjects and computed the mean power spectra of the corresponding TC. A set of ICs were identified as ICNs if peak activation fell on gray matter and showed overlap with known brain regions in the default mode network (DMN), sensorimotor network (SMN), salience network (SN), and central executive network (CEN) and exhibited primarily low frequency power. This selection procedure resulted in 30 ICNs out of the 80 ICs obtained. Compared to defining regions of interest based on anatomical brain atlases, the 
ICNs identified by group ICA are functionally homogeneous and may be better at capturing individual differences of real functional boundaries in the brain [14]. Additional postprocessing steps were performed on the time courses of selected ICNs, including: (1) detrending linear, quadratic, and cubic trends; (2) conducting multiple regressions of the 6 realignment parameters and their temporal derivatives; (3) despiking detected outliers; and (4) band-pass filtering with a cut-off frequency between 0.01 and $0.15 \mathrm{~Hz}$.

The FC matrix was constructed based on the time courses (TCs) of ICNs by calculating pairwise Pearson's correlations among TCs and z-transforming (Figure 1.2). Since 1) the connectivity matrix was symmetric, the lower and upper diagonal connectivity profiles were identical and 2) all diagonal values equaled 1 ( $i=j$ in Figure 1.2), we had $435(=30 \times 29 / 2)$ rsFCs across four networks for further investigation.

\section{Topological analysis}

After obtaining the functional connectivity matrices, graph theory approaches were applied to examine the abnormal topological organization of functional networks in cLBP patients (Figure 3.3). We defined 30 ICNs as nodes and the connectivity between each ICN as edges [50]. The analyses were performed using GRETNA toolbox (http://www.nitrc.org/projects/ gretna) [66]. Similar to previous studies [30,66,71], we first applied a sparsity threshold $S$ (the ratio of the number of actual edges to the maximum possible number of edges in a network) to all connectivity matrices for 0.1 to 0.5 with a step of 0.05 . After that, connectivity matrices were compared with random networks (100 times) to test whether they were configured with significantly non-random topology. This procedure resulted in an undirected and unweighted adjacency matrix for each subject (edges were designated as 1 if an edge between node $i$ and node $j$ was larger than the threshold we selected and 0 if it was smaller than the threshold; absolute values of connectivity were considered).

For brain networks at each sparsity threshold, we calculated three nodal metrics: degree, efficiency, and betweenness, as regional network measures. A detailed review of uses and interpretations of these measures can be found in [50]. In brief, the degree reflects the number of connections of a node within a network; efficiency measures the information propagation ability of the node with the rest of the nodes in the network; and betweenness captures the influence of a node over information flow between all other nodes in the network. All these three measures have been widely applied to study dysfunctional brain networks in patients $[30,55,71]$. To avoid the specific selection of a threshold, we applied an area under the curve (AUC) approach which is sensitive at detecting topological alteration of brain dysfunctions [31,71]. For each measure, the AUC was calculated within the sparsity range and compared between cLBP patients and HCs using a two sample t-test (control covariates: age, gender and BDI). Results were corrected using false discovery rate (FDR) with a corrected significance level of $\mathrm{p}_{\mathrm{FDR}}<0.05$.

\section{Multivariate pattern analysis}

To study abnormal rsFC across four networks in cLBP patients, we built a multivariate linear model with rsFC as an independent variable and group label (1 for cLBP and 0 for $\mathrm{HC}$ ) as a dependent variable (Figure 1.4). This model has been widely used in fMRI studies to 
identify brain patterns related to behavior and disease $[15,39,59,64,65]$. The model was decoded using a support vector classifier (SVC) implemented by LIBSVM [18], resulting in a pattern of classification weights across all rsFCs [57,58], and the significance of each rsFC in discriminating cLBP from HCs was assessed with bootstrap testing (see Statistical analyses for details).

To examine the extent to which rsFC captured functional abnormities in patients' brains, we also used all rsFCs as features to classify two cohorts of participants based on 5-fold cross validation, which ensures separation of training and testing samples [18]. In training, we fed training samples and their corresponding labels to the classifier, and the result was the classification weights for all features and a decision boundary for separating the two cohorts. In testing, the pattern of classification weights and decision boundary were applied to the test samples, yielding binary labels that constituted the predicted class for those samples (Figure 1.5). Accuracy, sensitivity, specificity and area under the receiver operating characteristics (ROC) curve were used to illustrate classification performance. We used permutation tests to statistically assess the performance of classification (see Statistical analyses for details).

\section{Correlation analysis}

In topological analysis and MVPA, we found distinct abnormalities in the ICN of medial prefrontal cortex/rostral anterior cingulate cortex (mPFC/rACC) and its rsFCs for cLBP patients compared to HCs (see Results for details), indicating that the mPFC/rACC might be a central hub in the abnormal rsFC of cLBP patients. Given the important role of mPFC/ rACC in pathophysiology of chronic pain $[8,10,37]$ and pain modulation $[34,68]$, we applied correlation analysis between $\mathrm{mPFC} / \mathrm{rACC}$ rsFCs and clinical symptoms.

Correlation significance was corrected for multiple comparisons between abnormal mPFC/ rACC $\operatorname{rsFCs}(N=14$, see Results for details) and each of the clinical symptoms (pain severity and duration) using $p_{\mathrm{FDR}}<0.05$. In addition, to further understand the relationship between abnormal rsFC and the affective, emotional and sensory aspects of chronic pain, we calculated the correlation between abnormal rsFCs and PROMIS subscores (see Clinical assessments for all 7 subscores). Results were corrected for multiple comparisons between abnormal mPFC rsFCs $(N=14)$ and each of the PROMIS subscores using $\mathrm{p}_{\mathrm{FDR}}<0.05$.

\section{Independent validation}

We proposed that if rsFC across the four networks captured functional brain abnormalities in cLBP patients, the SVC would be able to discriminate cLBP from HCs in an independent dataset. The SVC trained with the first dataset was applied to the independent sample of cLBP patients and HCs with no further model fitting. In addition, we calculated the correlation between abnormal rsFCs and clinical symptoms in the independent sample to test the validity of findings from the first dataset. The test was focused on the abnormal rsFCs showing a significant relationship with clinical symptoms in the first dataset. 


\section{Statistical analyses}

To threshold and select the most discriminative rsFC, we constructed 1000 bootstrap samples (with replacement) consisting of paired rsFCs and class labels and ran SVC on each. A one-sample t-test was performed for each rsFC based on the proportion of weights below or above zero and subjected to multiple comparisons.

In permutation testing, we randomly permuted data labels prior to training. Cross-validation was performed on the permuted dataset, and the procedure was repeated 10000 times. If the classifier trained on real data labels had an accuracy that exceeded the $95 \%$ confidence interval generated from the accuracies of the classifiers trained on randomly relabeled data labels, it was considered to be performing well.

\section{Results}

\section{Demographics of cLBP and HCs}

The first cohort consisted of 50 cLBP patients ( 31 females; age $39.5 \pm 13.0$, mean \pm standard deviation) and $44 \mathrm{HCs}$ ( 19 females; age $36.9 \pm 8.2$ ). A two-sample t-test and chi-square test showed no significant differences in age or gender ( $p=0.26$ and $p=0.07$, respectively). The validation cohort consisted of $30 \mathrm{cLBP}$ patients (14 females; age $35.0 \pm 9.0)$ and $30 \mathrm{HCs}$ (14 females; age $34.2 \pm 1.5)$. Statistical tests showed no significant differences in age ( $p=0.63)$ or gender $(p=0.61)$. The clinical characteristics of cLBP patients are summarized in Table 1. In this study, we used pain intensity to measure patients' pain perception before the MRI scan, while pain duration, pain severity score and PROMIS subscores were used to evaluate patients' daily experience beyond pain perception. Higher scores of pain interference, sleep disturbance, fatigue, anxiety, and depression and lower scores of physical function and social function indicated stronger disturbances in pain and related symptoms. According to the guideline and manual of the PROMIS system, 50 is the mean and 10 is the standard deviation for the US general population. Therefore, our cLBP patients only exhibited significantly comorbid symptoms in pain interference $(61.3 \pm 5.6)$ and physical function $(33.2 \pm 4.3)$. One patient in the first cohort was not included in the fMRI analyses due to excessive head movement.

\section{Spatial GICA and selected ICNs}

A total of 30 ICs were identified as ICNs since their activation peaks fell on gray matter and had low spatial overlap with known vascular, ventricular, motion, and other artifacts (Figure 2). These 30 ICNs were categorized into 4 resting-state networks, the DMN, SMN, SN, and $\mathrm{CEN}$, based on their functional meanings and anatomical information. A detailed spatial map of each ICN is shown in Supplementary Figure S1. Component labels and peak coordinates are provided in Supplementary Table S1.

\section{Abnormal topological property}

In the first cohort of subjects, we observed significantly abnormal FCs across four networks in cLBP patients (Figure 3A). Node degree, efficiency, and betweenness were significantly higher in the ICN of mPFC/rACC (a conjunction analysis, which compared the ICN with Automated Anatomical Labeling [AAL] atlas, showed that $53.6 \%$ of the ICN were located 
in $\mathrm{mPFC}, 33.2 \%$ of the ICN were located in $\mathrm{rACC}$, and the peak coordinate $[\mathrm{x}=-7 \mathrm{~mm}, \mathrm{y}=$ $41 \mathrm{~mm}, \mathrm{z}=-14 \mathrm{~mm}$ ] of the ICN located in mPFC) for cLBP patients compared to HCs, indicating that the mPFC/rACC might be a central hub in the abnormal rsFC of cLBP patients. Other regions did not show significant difference in all three metrics between cLBP and HCs. Detailed topological results of all 30 regions can be found in Supplementary Table S2.

\section{Abnormal mPFC / rACC connectivity in CLBP}

Figure 3B summaries abnormal mPFC/rACC connectivity in cLBP patients. The rsFCs between the $\mathrm{mPFC} / \mathrm{rACC}$ and posterior DMN regions (posterior cingulate cortex [PCC] and angular gyrus $[\mathrm{AG}]$ ) were decreased in cLBP patients, while $\mathrm{mPFC} / \mathrm{rACC}$ FCs with the SMN (postcentral gyrus [PoCG], superior parietal lobe [SPL] and paracentral lobe [ParaCL]) and SN (putamen, insula, anterior cingulate cortex [ACC] and caudate) were increased. Connections between the $\mathrm{mPFC} / \mathrm{rACC}$ and precentral (PreCG) and medial frontal gyri (MFG) in the CEN were decreased, while the connection between the mPFC/rACC and middle frontal gyrus (MiFG) was increased. Correlation analyses (adjusted for age, gender and BDI score; Figure 3C) showed that rsFC between the $\mathrm{mPFC} / \mathrm{rACC}$ and $\mathrm{ACC}$ was correlated with duration of $\operatorname{cLBP}\left(\mathrm{r}=-0.32, p_{F D R}=0.03\right)$ and $\mathrm{rsFC}$ between the $\mathrm{mPFC} /$ $\mathrm{rACC}$ and PCC/AG was correlated with pain severity $\left(\mathrm{r}=-0.33, p_{F D R}=0.02\right)$. It is worth noting that abnormal rsFCs in cLBP patients were not correlated with their pain intensity.

The association between abnormal rsFC and PROMIS subscores were shown in Figure 4A. Among all physical, mental and social disability scores, pain interference was correlated with $\mathrm{rsFC}$ between the $\mathrm{mPFC} / \mathrm{rACC}$ and PoCG $\left(\mathrm{r}=-0.43, p_{F D R}=0.002\right)$. Sleep disturbance was correlated with $\mathrm{rsFC}$ between the $\mathrm{mPFC} / \mathrm{rACC}$ and caudate $\left(\mathrm{r}=-0.37, p_{F D R}=0.009\right)$, but in general the sleep score $(51.6 \pm 3.4)$ indicated that cLBP patients did not have sleep disorders.

In the validation dataset (Figure 4B and Figure 5), we also found decreased mPFC/rACC rsFC with the DMN (PCC, AG) and CEN (PreCG and MFG) and increased mPFC/rACC FC with the SN (putamen, ACC and caudate). These results are congruent with findings from the first dataset. In particular, we observed significant correlations between $\mathrm{mPFC} / \mathrm{rACC}$ ACC $\mathrm{rsFC}$ and duration of $\mathrm{cLBP}(\mathrm{r}=-0.37, p=0.04)$ and between $\mathrm{mPFC} / \mathrm{rACC}-\mathrm{PCC} / \mathrm{AG}$ rsFC and pain severity $(\mathrm{r}=-0.35, p=0.04)$. We also validated the significant relationship between the abnormal mPFC/rACC-PoCG $\mathrm{rsFC}$ and pain interference $(\mathrm{r}=-0.41, p=0.02)$ and a trend between the abnormal mPFC/rACC-Caudate $\mathrm{rsFC}$ and sleep disturbance $(\mathrm{r}=$ $-0.32, p=0.08)$.

\section{Internal cross-validated classification of cLBP and HC}

In the 5-fold cross-validation test, rsFC across the four networks discriminated cLBP from HCs and obtained a classification accuracy of $91.4 \%(p<0.001$; Figure 5A) with a sensitivity of $93.2 \%$ and a specificity of $89.8 \%$. An ROC curve was produced to further estimate the performance of the classification. The area under the ROC curve (AUC) equaled 0.96 , indicating a satisfactory classification ability (Figure 5B). The classification weights, 
indexed by the $t$ values (from bootstrap testing) for all rsFCs, are given in Supplementary Figure S5.

\section{External-validated classification of CLBP and HC}

The SVC obtained from the first dataset was used to discriminate cLBP patients from HCs in an independent dataset, obtaining an accuracy of $78.3 \%(p=0.001$; Figure $5 \mathrm{C})$ with a sensitivity of $80.0 \%$ and a specificity of $76.7 \%$. The AUC was 0.81 (Figure 5D).

\section{Discussion}

In this study, we investigated abnormal rsFC across four resting-state networks in cLBP patients and studied the relationships between abnormal rsFCs and clinical scores in cLBP. Using graph theory and MVPA, we found (1) the mPFC/rACC is the key node in the abnormal functional brain network of cLBP patients; (2) the rsFCs between the mPFC/rACC and other regions in the DMN were decreased, while $\mathrm{mPF} / \mathrm{rACC}-\mathrm{SMN}$ and $\mathrm{mPFC} / \mathrm{rACC}-\mathrm{SN}$ connectivities were increased; (3) abnormal $\mathrm{mPFC} / \mathrm{rACC}$ rsFCs were associated with duration, pain severity, and pain interference of cLBP but not correlated with pain intensity; and (4) the rsFCs in these four networks were able to achieve a cross-validation accuracy of $91 \%$ and an independent validation accuracy of $78 \%$ in discriminating cLBP patients from HCs. Thus, we believe the mPFC/rACC may serve as an important region for linking the DMN to the other three networks for cLBP patients and found that disrupted FCs were correlated with daily experience but not with pain perception.

Brain regions integrate and distribute information in powerful ways through several, interconnected networks. These regions can be defined as nodes with many edges, which places them in central positions for facilitating information transfer in networks. Previous studies found well-connected nodes (high-degree nodes) in the DMN, which is a collection of brain regions that are implicated in various 'high-level' cognitive processes [13,48]. In our study, the ICN of mPFC/rACC had a significantly higher node degree, efficiency, and betweenness in cLBP patients than in HCs, highlighting its potential role in the functional reorganization of brain networks in chronic pain. The $\mathrm{mPFC}$ is a region involving multiple brain functions, such as pain modulation and emotional evaluation $[9,68]$. Baliki and colleagues reported an enhanced high-frequency fluctuation of fMRI signals in cLBP patients primarily mapped to the $\mathrm{mPFC}$ and brain regions within the DMN, and chronic pain patients showed decreased connectivity of the $\mathrm{MPFC}$ with the posterior parts of the DMN $[8,10]$ but increased connectivity between $\mathrm{mPFC}$ and nucleus accumbens (NAc) in the reward system [11]. Similar findings have also been reported in animal studies, indicating the important role of the prefrontal cortex and its projections to the NAc in regulating affective and motivational components of pain $[19,38,49]$. In line with these studies, we found that abnormal mPFC-PCC/AG connectivity was correlated with the severity (bothersomeness) of chronic pain but not pain intensity, further demonstrating that cLBP may modulate brain functions beyond the pain system itself in ways that may be maladaptive, affecting patients' daily experience.

We found that cLBP patients showed higher mPFC-SN connectivity compared to HCs. The $\mathrm{SN}$ detects relevant stimuli from the environment and coordinates other brain networks to 
generate behavioral responses [60], while the DMN is involved in higher-level thought. In $\mathrm{HCs}$, the activity between the DMN and $\mathrm{SN}$ is anticorrelated. The $\mathrm{SN}$ is active and the DMN suppressed when attention is engaged, while the reverse is true when the brain is engaged in internal thought [24]. Unlike acute pain, however, in which attention to sudden, external pain activates the SN and suppresses the DMN, chronic pain may cause the patient to think more about their constant pain, activating the DMN. Thus, disruption in DMN-SN FC can be found in several chronic pain disorders [9,27]. In our study, we found that connectivity between the mPFC and ACC in the SN was correlated with the duration of cLBP, suggesting that DMN-SN abnormalities may not be specific to pain. Rather, the continuous experience of salient symptoms may become ingrained in internal thought and alter attention. In addition, we found increased mPFC-SMN connectivity, indicating that communication between the mPFC and sensory-related regions was also altered in cLBP patients.

It is interesting to note that abnormal rsFCs in cLBP patients were associated with the severity of chronic pain and pain interference, but not with pain intensity. These results were consistent with a previous study suggesting that chronic pain encompasses more than just the feeling of pain [9], relating to symptoms that significantly diminish quality of life (e.g., physical disability, pain interference) [5].

There are several potential confounders to be noticed. First, previous studies have suggested cLBP patients have a high prevalence of depression, and depression may induce abnormal FC between the mPFC and DMN. In our study, cLBP patients had PROMIS-depression and BDI scores within the normal range. Since PROMIS scores were not recorded for HCs, we included BDI scores as a covariate in between-group analyses (e.g., MVPA, topological analysis) and within-group analysis (e.g., correlation between abnormal rsFC and cLBP pain severity). We also performed a correlation analysis between abnormal rsFC and PROMISdepression score and did not find any significant relationship. These results at least partly minimized the effects of depression. Second, cLBP patients may have different levels of vigilance compared to HCs. Unlike other artifacts (e.g., noise, head motion), rsFC changes related to changes in vigilance are physiologically meaningful. PROMIS-sleep disturbance scores showed cLBP patients overall did not have difficulty in sleep during their life. We also examined the level of vigilance between the two groups of subjects using wellestablished rsFC signatures of vigilance[53], and results showed no systematic differences between cLBP and HCs (Supplementary Material). Future studies should include cardiac, respiratory, or eye-tracking data[3] to further characterize the pathophysiology of cLBP patients.

MVPA and machine learning techniques have been widely applied in translational neuroimaging studies to provide a basis for identifying neuropathological features of different diseases and to show potential clinical utility beyond current clinical diagnostic categories $[1,6,20,29,45,46,67,70]$. For instance, investigators have applied this method to identify brain signatures for neurological and psychiatric diseases, such as Alzheimer's disease and dementia [32], Parkinson's disease [54], major depression [70], schizophrenia [51], and ADHD [25]. Most of these studies have focused on identifying brain signatures for discriminating patients from HCs and consequently establishing a meaningful neurophysiological basis for disorders of interest. In a previous study with a similar sample 
size to our study [61], investigators applied an SVM model using abnormal gray matter density to classify cLBP patients $(n=47)$ and HCs $(n=47)$, achieving an accuracy of $76 \%$. In our study using resting-state fMRI data, we achieved an accuracy of $91 \%$, which was validated with an independent dataset with $78 \%$ accuracy. We therefore demonstrated the feasibility and reliability of our proposed model for identifying abnormal functional and structural patterns of the brain processing spontaneous pain.

There are several limitations to this study. First, all data included in the analysis used the same MRI scanner and population. Future studies are needed to further clarify the roles of different variables such as MRI scanner and parameters [2]. Second, the relationship between functional and anatomical abnormalities in cLBP patients is still not clear. Further investigations using multi-modality fusion techniques may provide insight. Third, it would be interesting to apply the model to other types of chronic pain disorders such as fibromyalgia and migraine to study the specific and shared neural mechanisms of different chronic pain conditions.

In conclusion, we found abnormal FCs between the MPFC and other brain regions across the DMN, SMN, SN and CEN in cLBP patients. The abnormal FCs were correlated with pain severity, pain interference and sleep disturbance in patients. Using an MVPA approach, we were able to discriminate cLBP patients from matched HCs with more than $91 \%$ accuracy in the first cohort and $78 \%$ accuracy in the validation cohort. The identification of abnormal functional dynamics in cLBP patients may lead to a better understanding of the disorder, better-targeted treatment, and the development of diagnostic and prognostic indicators.

\section{Supplementary Material}

Refer to Web version on PubMed Central for supplementary material.

\section{Acknowledgement}

This study is supported by R21AT008707, R61AT009310, R01AT008563 from NIH/NCCIH.

\section{References}

[1]. Abi-Dargham A, Horga G. The search for imaging biomarkers in psychiatric disorders. Nat. Med 2016;22:1248-1255. [PubMed: 27783066]

[2]. Abraham A, Milham MP, Di Martino A, Craddock RC, Samaras D, Thirion B, Varoquaux G. Deriving reproducible biomarkers from multi-site resting-state data: An Autism-based example. Neuroimage 2017;147:736-745. [PubMed: 27865923]

[3]. Allen EA, Damaraju E, Eichele T, Wu L, Calhoun VD. EEG Signatures of Dynamic Functional Network Connectivity States. Brain Topogr. 2018;31:101-116. [PubMed: 28229308]

[4]. Apkarian AV, Baliki MN, Geha PY. Towards a theory of chronic pain. Prog. Neurobiol 2009;87:81-97. [PubMed: 18952143]

[5]. Apkarian VA, Sosa Y, Krauss BR, Thomas SP, Fredrickson BE, Levy RE, Harden NR, Chialvo DR. Chronic pain patients are impaired on an emotional decision-making task. Pain 2004;108:129-136. [PubMed: 15109516]

[6]. Arribas JI, Calhoun VD, Adali T. Automatic Bayesian Classification of Healthy Controls, Bipolar Disorder, and Schizophrenia Using Intrinsic Connectivity Maps From fMRI Data. IEEE Trans. Biomed. Eng. 2010;57:2850-2860. [PubMed: 20876002] 
[7]. Ashburner J, Friston K. Unified segmentation. Neuroimage 2005;26:839-851. [PubMed: 15955494]

[8]. Baliki MN, Baria AT, Apkarian AV. The Cortical Rhythms of Chronic Back Pain. J. Neurosci 2011;31:13981-13990. [PubMed: 21957259]

[9]. Baliki MN, Geha PY, Apkarian AV, Chialvo DR. Beyond feeling: chronic pain hurts the brain, disrupting the default-mode network dynamics. J. Neurosci 2008;28:1398-403. [PubMed: 18256259]

[10]. Baliki MN, Mansour AR, Baria AT, Apkarian AV. Functional Reorganization of the Default Mode Network across Chronic Pain Conditions. PLoS One 2014;9:e106133. [PubMed: 25180885]

[11]. Baliki MN, Petre B, Torbey S, Herrmann KM, Huang L, Schnitzer TJ, Fields HL, Apkarian AV. Corticostriatal functional connectivity predicts transition to chronic back pain. Nat. Neurosci 2012;15:1117-9. [PubMed: 22751038]

[12]. Borsook D, Edwards R, Elman I, Becerra L, Levine J. Pain and analgesia: the value of salience circuits. Prog. Neurobiol 2013;104:93-105. [PubMed: 23499729]

[13]. Buckner RL, Sepulcre J, Talukdar T, Krienen FM, Liu H, Hedden T, Andrews-Hanna JR, Sperling RA, Johnson KA. Cortical Hubs Revealed by Intrinsic Functional Connectivity: Mapping, Assessment of Stability, and Relation to Alzheimer's Disease. J. Neurosci 2009;29:1860-1873. [PubMed: 19211893]

[14]. Calhoun VD, Adali T, Pearlson GD, Pekar JJ. A method for making group inferences from functional MRI data using independent component analysis. Hum. Brain Mapp 2001;14:140 151. [PubMed: 11559959]

[15]. Cao J, Tu Y, Orr SP, Lang C, Park J, Vangel M, Chen L, Gollub R, Kong J. Analgesic Effects Evoked by Real and Imagined Acupuncture: A Neuroimaging Study. Cereb. Cortex 2018.

[16]. Cella D, Riley W, Stone A, Rothrock N, Reeve B, Yount S, Amtmann D, Bode R, Buysse D, Choi S, Cook K, Devellis R, DeWalt D, Fries JF, Gershon R, Hahn EA, Lai J-S, Pilkonis P, Revicki D, Rose M, Weinfurt K, Hays R, PROMIS Cooperative Group. The Patient-Reported Outcomes Measurement Information System (PROMIS) developed and tested its first wave of adult selfreported health outcome item banks: 2005-2008. J. Clin. Epidemiol 2010;63:1179-94. [PubMed: 20685078]

[17]. Cella D, Yount S, Rothrock N, Gershon R, Cook K, Reeve B, Ader D, Fries JF, Bruce B, Rose M, PROMIS Cooperative Group. The Patient-Reported Outcomes Measurement Information System (PROMIS): progress of an NIH Roadmap cooperative group during its first two years. Med. Care 2007;45:S3-S11.

[18]. Chang C, Lin C. LIBSVM: a library for support vector machines. ACM Trans. Intell. Syst. Technol 2011;2:27.

[19]. Chang P-C, Centeno MV, Procissi D, Baria A, Apkarian AV. Brain activity for tactile allodynia. Pain 2017;158:488-497. [PubMed: 28135213]

[20]. Chen J, Wang Z, Tu Y, Liu X, Jorgenson K, Ye G, Lin C, Liu J, Park J, Lang C, Liu B, Kong J. Regional Homogeneity and Multivariate Pattern Analysis of Cervical Spondylosis Neck Pain and the Modulation Effect of Treatment. Front. Neurosci 2018;12:900. [PubMed: 30574062]

[21]. Ciric R, Wolf DH, Power JD, Roalf DR, Baum GL, Ruparel K, Shinohara RT, Elliott MA, Eickhoff SB, Davatzikos C, Gur RC, Gur RE, Bassett DS, Satterthwaite TD. Benchmarking of participant-level confound regression strategies for the control of motion artifact in studies of functional connectivity. Neuroimage 2017;154:174-187. [PubMed: 28302591]

[22]. Farmer MA, Baliki MN, Apkarian AV. A dynamic network perspective of chronic pain. Neurosci. Lett 2012;520:197-203. [PubMed: 22579823]

[23]. Glass JM, Williams DA, Fernandez-Sanchez M-L, Kairys A, Barjola P, Heitzeg MM, Clauw DJ, Schmidt-Wilcke T. Executive function in chronic pain patients and healthy controls: different cortical activation during response inhibition in fibromyalgia. J. Pain 2011;12:1219-29. [PubMed: 21945593]

[24]. Goulden N, Khusnulina A, Davis NJ, Bracewell RM, Bokde AL, McNulty JP, Mullins PG. The salience network is responsible for switching between the default mode network and the central executive network: Replication from DCM. Neuroimage 2014;99:180-190. [PubMed: 24862074] 
[25]. Hart H, Chantiluke K, Cubillo AI, Smith AB, Simmons A, Brammer MJ, Marquand AF, Rubia K. Pattern classification of response inhibition in ADHD: Toward the development of neurobiological markers for ADHD. Hum. Brain Mapp 2014;35:3083-3094. [PubMed: 24123508]

[26]. Hart LG, Deyo RA, Cherkin DC. Physician office visits for low back pain. Frequency, clinical evaluation, and treatment patterns from a U.S. national survey. Spine. 1995;20:11-9. [PubMed: 7709270]

[27]. Hemington KS, Wu Q, Kucyi A, Inman RD, Davis KD. Abnormal cross-network functional connectivity in chronic pain and its association with clinical symptoms. Brain Struct. Funct 2016;221:4203-4219. [PubMed: 26669874]

[28]. Himberg J, Hyvarinen A. Icasso: software for investigating the reliability of ICA estimates by clustering and visualization. 2003 IEEE XIII Workshop on Neural Networks for Signal Processing pp. 259-268.

[29]. Jung M, Tu Y, Park J, Jorgenson K, Lang C, Song W, Kong J. Surface-based shared and distinct resting functional connectivity in attention-deficit hyperactivity disorder and autism spectrum disorder. Br. J. Psychiatry 2018:1-6.

[30]. Keown CL, Datko MC, Chen CP, Maximo JO, Jahedi A, Müller R-A. Network Organization Is Globally Atypical in Autism: A Graph Theory Study of Intrinsic Functional Connectivity. Biol. Psychiatry Cogn. Neurosci. Neuroimaging 2017;2:66-75. [PubMed: 28944305]

[31]. Kim J, Criaud M, Cho SS, Díez-Cirarda M, Mihaescu A, Coakeley S, Ghadery C, Valli M, Jacobs MF, Houle S, Strafella AP. Abnormal intrinsic brain functional network dynamics in Parkinson's disease. Brain 2017;140:2955-2967. [PubMed: 29053835]

[32]. Kippenhan JS, Barker WW, Pascal S, Nagel J, Duara R. Evaluation of a neural-network classifier for PET scans of normal and Alzheimer's disease subjects. J. Nucl. Med 1992;33:1459-67. [PubMed: 1634935]

[33]. Kong J, Spaeth RB, Wey H-Y, Cheetham A, Cook AH, Jensen K, Tan Y, Liu H, Wang D, Loggia ML, Napadow V, Smoller JW, Wasan AD, Gollub RL. S1 is associated with chronic low back pain: a functional and structural MRI study. Mol. Pain 2013;9:43. [PubMed: 23965184]

[34]. Kong J, Wang Z, Leiser J, Minicucci D, Edwards R, Kirsch I, Wasan AD, Lang C, Gerber J, Yu S, Napadow V, Kaptchuk TJ, Gollub RL. Enhancing treatment of osteoarthritis knee pain by boosting expectancy: A functional neuroimaging study. Neuro Image Clin. 2018;18:325-334.

[35]. Kucyi A, Davis KD. The dynamic pain connectome. Trends Neurosci. 2015;38:86-95. [PubMed: 25541287]

[36]. Kucyi A, Davis KD. The Neural Code for Pain: From Single-Cell Electrophysiology to the Dynamic Pain Connectome. Neurosci. 2017;23:397-414.

[37]. Kucyi A, Moayedi M, Weissman-Fogel I, Goldberg MB, Freeman BV, Tenenbaum HC, Davis KD. Enhanced Medial Prefrontal-Default Mode Network Functional Connectivity in Chronic Pain and Its Association with Pain Rumination. J. Neurosci. 2014;34:3969-3975. [PubMed: 24623774]

[38]. Lee M, Manders TR, Eberle SE, Su C, D'amour J, Yang R, Lin HY, Deisseroth K, Froemke RC, Wang J. Activation of Corticostriatal Circuitry Relieves Chronic Neuropathic Pain. J. Neurosci. 2015;35:5247-5259. [PubMed: 25834050]

[39]. Lindquist MA, Krishnan A, Lopez-Sol M, Jepma M, Woo CW, Koban L, Roy M, Atlas LY, Schmidt L, Chang LJ, Reynolds Losin EA, Eisenbarth H, Ashar YK, Delk E, Wager TD. Groupregularized individual prediction: Theory and application to pain. Neuroimage 2016.

[40]. Loggia ML, Kim J, Gollub RL, Vangel MG, Kirsch I, Kong J, Wasan AD, Napadow V. Default mode network connectivity encodes clinical pain: an arterial spin labeling study. Pain 2013;154:24-33. [PubMed: 23111164]

[41]. Mao J Current challenges in translational pain research. Trends Pharmacol. Sci. 2012;33:568573. [PubMed: 22959652]

[42]. Martucci KT, Mackey SC. Neuroimaging of Pain. Anesthesiology 2018;128:1241-1254. [PubMed: 29494401] 
[43]. Murphy K, Birn RM, Handwerker DA, Jones TB, Bandettini PA. The impact of global signal regression on resting state correlations: Are anti-correlated networks introduced? Neuroimage 2009;44:893-905. [PubMed: 18976716]

[44]. Napadow V, LaCount L, Park K, As-Sanie S, Clauw DJ, Harris RE. Intrinsic brain connectivity in fibromyalgia is associated with chronic pain intensity. Arthritis Rheum. 2010;62:2545-2555. [PubMed: 20506181]

[45]. Orrù G, Pettersson-Yeo W, Marquand AF, Sartori G, Mechelli A. Using Support Vector Machine to identify imaging biomarkers of neurological and psychiatric disease: A critical review. Neurosci. Biobehav. Rev 2012;36:1140-1152. [PubMed: 22305994]

[46]. Pereira F, Mitchell T, Botvinick M. Machine learning classifiers and fMRI: a tutorial overview. Neuroimage 2009;45:S199-S209. [PubMed: 19070668]

[47]. Power JD, Barnes KA, Snyder AZ, Schlaggar BL, Petersen SE. Spurious but systematic correlations in functional connectivity MRI networks arise from subject motion. Neuroimage 2012;59:2142-2154. [PubMed: 22019881]

[48]. Power JD, Schlaggar BL, Lessov-Schlaggar CN, Petersen SE. Evidence for hubs in human functional brain networks. Neuron 2013;79:798-813. [PubMed: 23972601]

[49]. Ren W, Centeno MV, Berger S, Wu Y, Na X, Liu X, Kondapalli J, Apkarian AV, Martina M, Surmeier DJ. The indirect pathway of the nucleus accumbens shell amplifies neuropathic pain. Nat. Neurosci. 2016;19:220-222. [PubMed: 26691834]

[50]. Rubinov M, Sporns O. Complex network measures of brain connectivity: Uses and interpretations. Neuroimage 2010;52:1059-1069. [PubMed: 19819337]

[51]. Shen H, Wang L, Liu Y, Hu D. Discriminative analysis of resting-state functional connectivity patterns of schizophrenia using low dimensional embedding of fMRI. Neuroimage 2010;49:3110-3121. [PubMed: 19931396]

[52]. Sherman KJ, Cherkin DC. Developing methods for acupuncture research: rationale for and design of a pilot study evaluating the efficacy of acupuncture for chronic low back pain. Altern. Ther. Health Med 2003;9:54-60. [PubMed: 14526711]

[53]. Tagliazucchi E, von Wegner F, Morzelewski A, Borisov S, Jahnke K, Laufs H. Automatic sleep staging using fMRI functional connectivity data. Neuroimage 2012;63:63-72. [PubMed: 22743197]

[54]. Tang CC, Poston KL, Eckert T, Feigin A, Frucht S, Gudesblatt M, Dhawan V, Lesser M, Vonsattel J-P, Fahn S, Eidelberg D. Differential diagnosis of parkinsonism: a metabolic imaging study using pattern analysis. Lancet Neurol. 2010;9:149-158. [PubMed: 20061183]

[55]. Termenon M, Jaillard A, Delon-Martin C, Achard S. Reliability of graph analysis of resting state fMRI using test-retest dataset from the Human Connectome Project. Neuroimage 2016;142:172187. [PubMed: 27282475]

[56]. Tracey I, Bushnell MC. How Neuroimaging Studies Have Challenged Us to Rethink: Is Chronic Pain a Disease? J. Pain 2009;10:1113-1120. [PubMed: 19878862]

[57]. Tu Y, Fu Z, Tan A, Huang G, Hu L, Hung YS, Zhang Z. A novel and effective fMRI decoding approach based on sliced inverse regression and its application to pain prediction. Neurocomputing 2018;273:373-384.

[58]. Tu Y, Tan A, Bai Y, Hung YS, Zhang Z. Decoding Subjective Intensity of Nociceptive Pain from Pre-stimulus and Post-stimulus Brain Activities. Front. Comput. Neurosci 2016;10:32. [PubMed: 27148029]

[59]. Tu Y, Zhang Z, Tan A, Peng W, Hung YS, Moayedi M, Iannetti GD, Hu L. Alpha and gamma oscillation amplitudes synergistically predict the perception of forthcoming nociceptive stimuli. Hum. Brain Mapp 2016;37:501-514. [PubMed: 26523484]

[60]. Uddin LQ. Salience processing and insular cortical function and dysfunction. Nat. Rev. Neurosci 2015;16:55-61. [PubMed: 25406711]

[61]. Ung H, Brown JE, Johnson KA, Younger J, Hush J, Mackey S. Multivariate classification of structural MRI data detects chronic low back pain. Cereb. Cortex 2014;24:1037-1044. [PubMed: 23246778] 
[62]. Vos de Wael R, Hyder F, Thompson GJ. Effects of Tissue-Specific Functional Magnetic Resonance Imaging Signal Regression on Resting-State Functional Connectivity. Brain Connect. 2017;7:482-490. [PubMed: 28825320]

[63]. Vos T, Flaxman A, Naghavi M, Lozano R, Michaud C. Years lived with disability (YLDs) for 1160 sequelae of 289 diseases and injuries 1990-2010: a systematic analysis for the Global Burden of Disease Study 2010. Lancet 2013;380:2163-2196.

[64]. Wager TD, Atlas LY, Leotti LA, Rilling JK. Predicting individual differences in placebo analgesia: contributions of brain activity during anticipation and pain experience. J. Neurosci. 2011;31:439-452. [PubMed: 21228154]

[65]. Wager TD, Atlas LY, Lindquist M a, Roy M, Woo C-W, Kross E. An fMRI-based neurologic signature of physical pain. N. Engl. J. Med. 2013;368:1388-97. [PubMed: 23574118]

[66]. Wang J, Wang X, Xia M, Liao X, Evans A, He Y. GRETNA: a graph theoretical network analysis toolbox for imaging connectomics. Front. Hum. Neurosci 2015;9:386. [PubMed: 26175682]

[67]. Woo C-W, Chang LJ, Lindquist MA, Wager TD. Building better biomarkers: Brain models in translational neuroimaging. Nat. Neurosci. Rev 2017;20:365-377.

[68]. Yu R, Gollub RL, Spaeth R, Napadow V, Wasan A, Kong J. Disrupted functional connectivity of the periaqueductal gray in chronic low back pain. Neuro Image Clin. 2014;6:100-108.

[69]. Yuan J, Purepong N, Kerr DP, Park J, Bradbury I, McDonough S. Effectiveness of Acupuncture for Low Back Pain. Spine. 2008;33:E887-E900. [PubMed: 18978583]

[70]. Zeng LL, Shen H, Liu L, Wang L, Li B, Fang P, Zhou Z, Li Y, Hu D. Identifying major depression using whole-brain functional connectivity: A multivariate pattern analysis. Brain 2012;135:1498-1507. [PubMed: 22418737]

[71]. Zhang J, Wang J, Wu Q, Kuang W, Huang X, He Y, Gong Q. Disrupted Brain Connectivity Networks in Drug-Naive, First-Episode Major Depressive Disorder. Biol. Psychiatry 2011;70:334-342. [PubMed: 21791259] 
1) Perform group ICA and identify ICNs

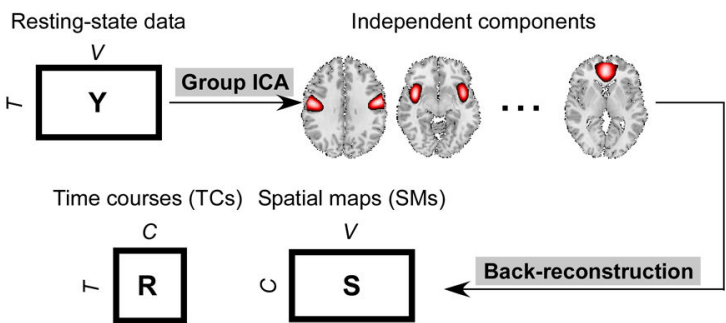

2) Construct connectivity matrix

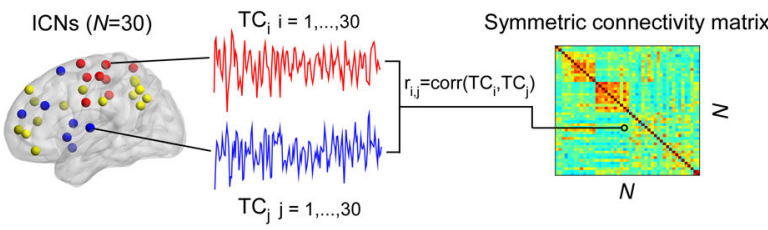

3) Topological analysis

Connectivity matrix

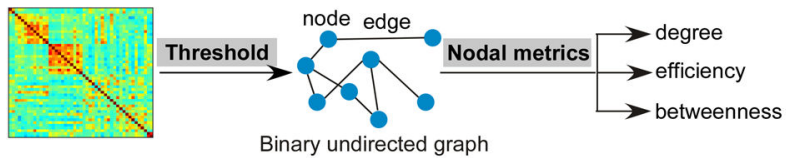

4) Multivariate pattern analysis

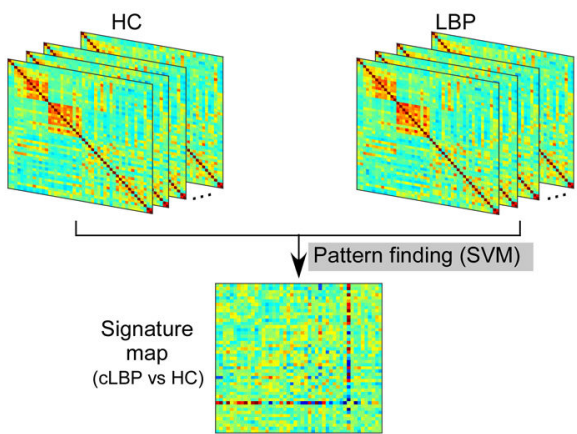

5) Classify cLBP patients
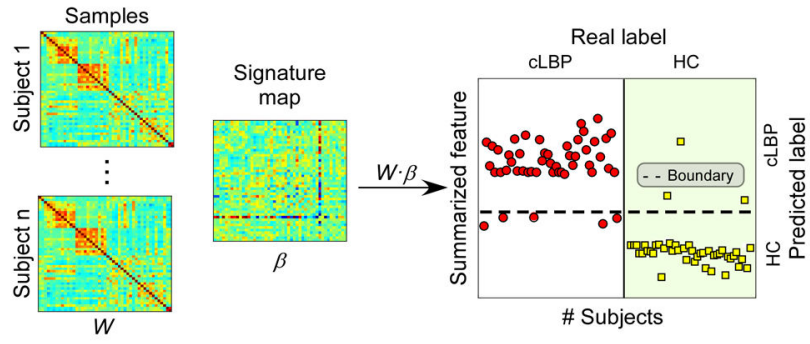

Figure 1.

Analysis flowchart to study abnormal functional connectivity in cLBP patients. Five major steps were included: 1) perform group independent component analysis (GICA) and select intrinsic connectivity networks (ICNs); 2) contruct connectivity matrix; 3) topological analysis; 4) multivariate pattern analysis (MVPA) and 5) classify cLBP and HCs. 

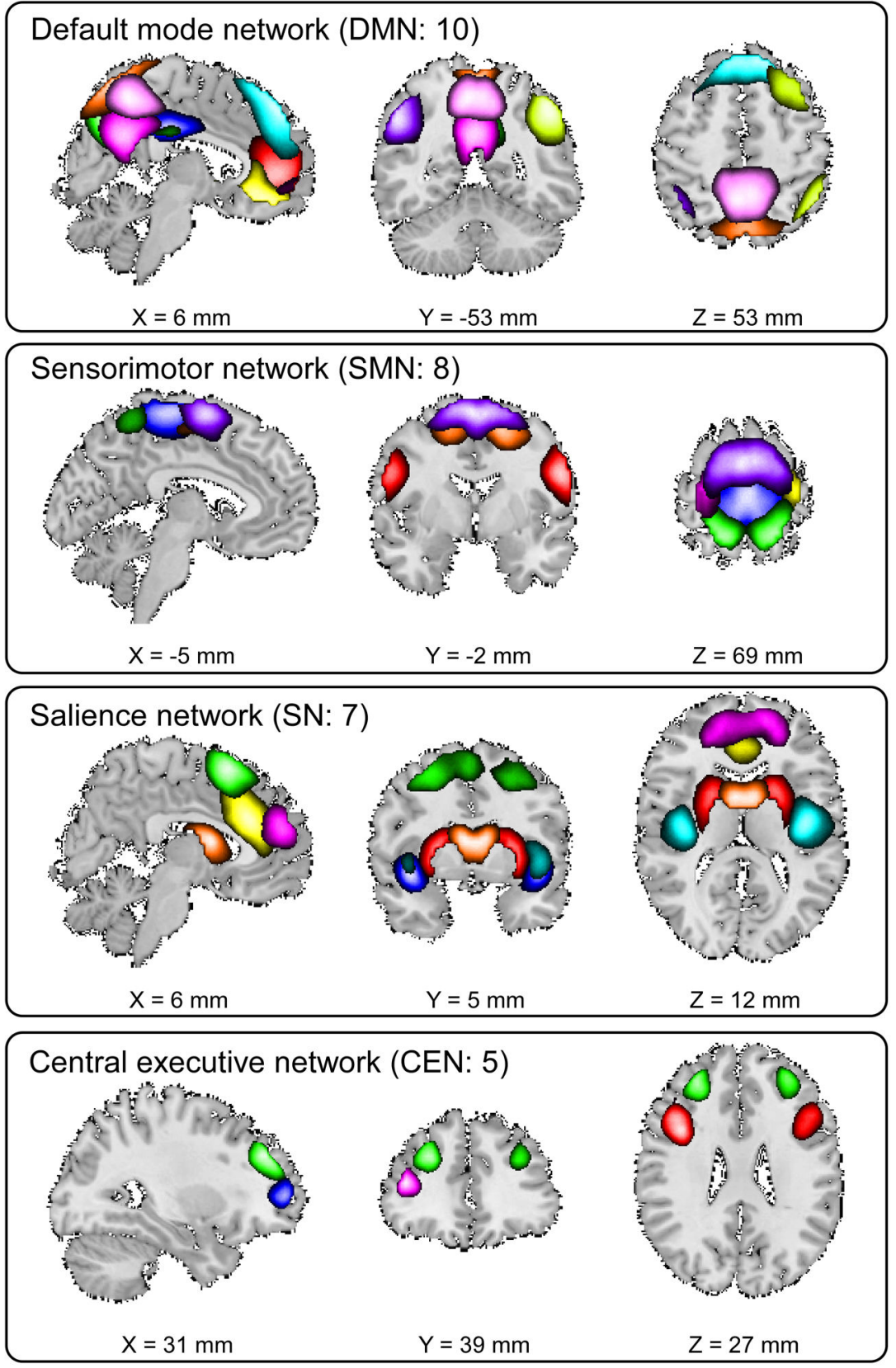

Figure 2.

Spatial maps of the 30 selected ICNs, sorted into four networks. Each color in the spatial maps corresponds to a different ICN. 


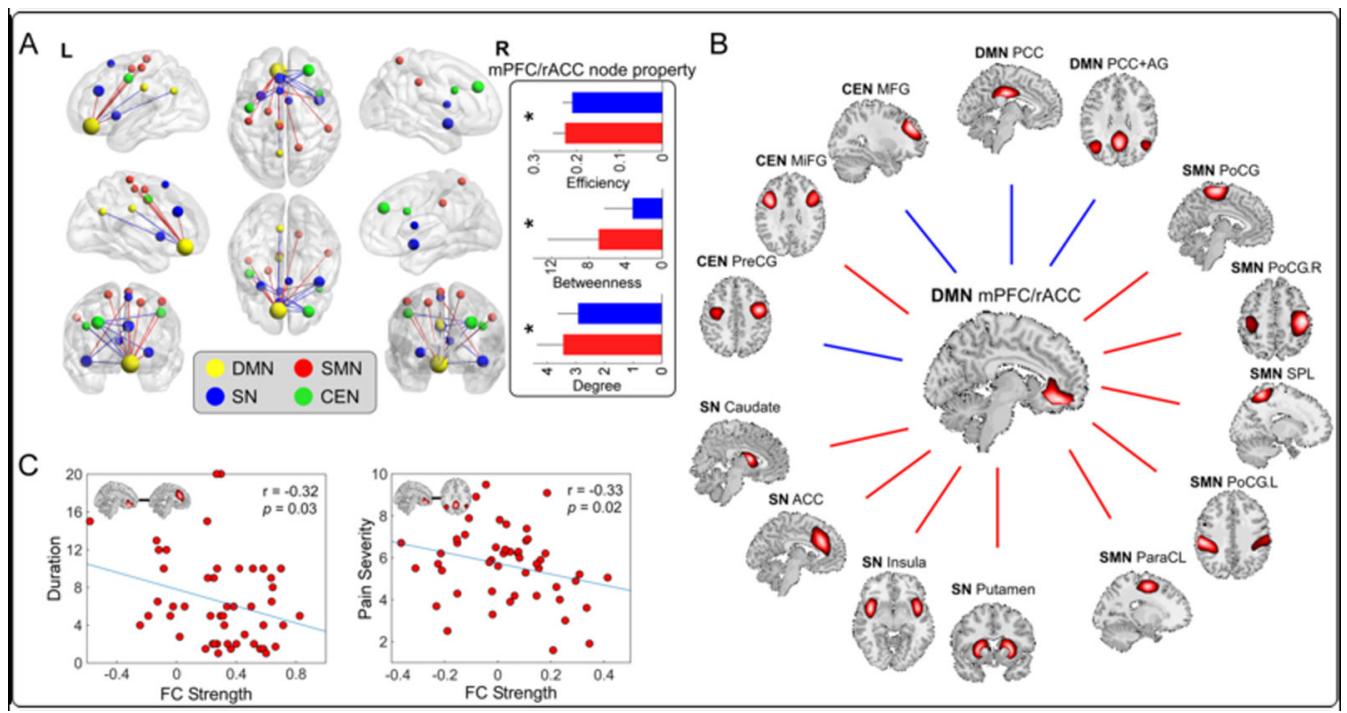

Figure 3.

Abnormal rsFC in cLBP patients. Panel A: MVPA identified abnormal FCs across four networks. A graph-theory-based measure showed cLBP patients had significantly higher $\mathrm{mPFC} / \mathrm{rACC}$ node degree, efficiency, and betweenness than HCs. Panel B: abnormal mPFC/ rACC FCs with other regions in cLBP patients. We found decreased mPFC/rACC-DMN connectivity and increased mPFC/rACC-SMN and mPFC/rACC-SN connectivity in cLBP. Panel $\mathrm{C}$ : $\mathrm{mPFC} / \mathrm{rACC}-\mathrm{ACC}$ rsFC was correlated with the duration of cLBP, and $\mathrm{mPFC} /$ rACC-PCC/AG rsFC was correlated with the severity of cLBP. PCC: posterior cingulate cortex; AG: angular gyrus; PoCG: post-central gyrus; SPL: superior parietal lobe; ParaCL: paracentral gyrus; ACC: anterior cingulate cortex; PreCG: precentral gyrus; MiFG: middle frontal gyrus; MFG: medial frontal gyrus. Red lines and blue lines in B represent increased and decreased rsFC in cLBP patients. 

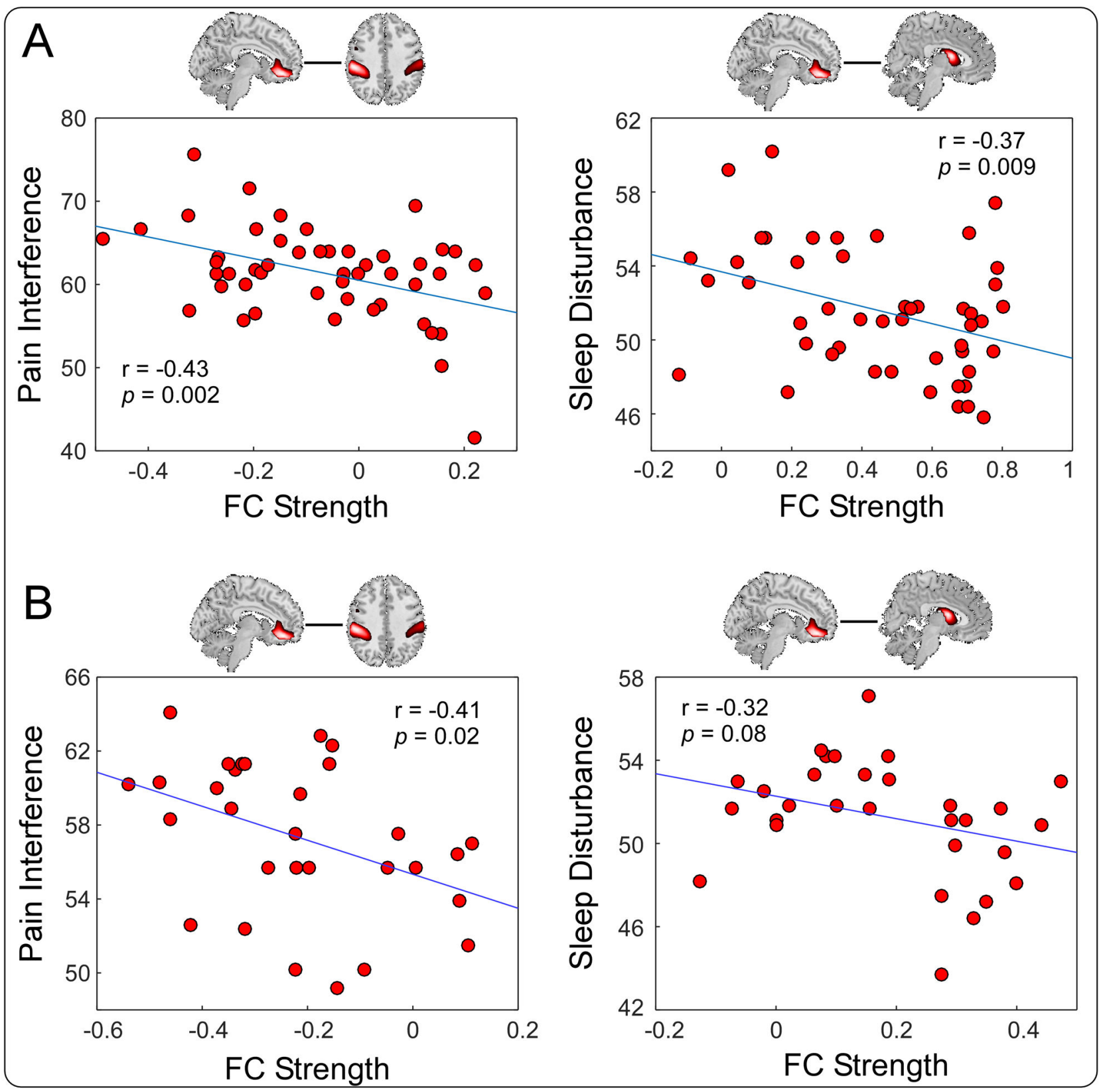

Figure 4.

Association between abnormal rsFC and PROMIS subscores. Panel A: Results from the first cohort. rsFC between the mPFC/rACC and PoCG was significantly correlated with pain interference, and rsFC between the $\mathrm{mPFC} / \mathrm{rACC}$ and caudate was significantly correlated with sleep disturbance. Panel B: Results from the independent cohort. rsFC between the $\mathrm{mPFC} / \mathrm{rACC}$ and PoCG was significantly correlated with pain interference, while rsFC between the $\mathrm{mPFC} / \mathrm{rACC}$ and caudate showed a non-significant trend towards correlating with sleep disturbance. 


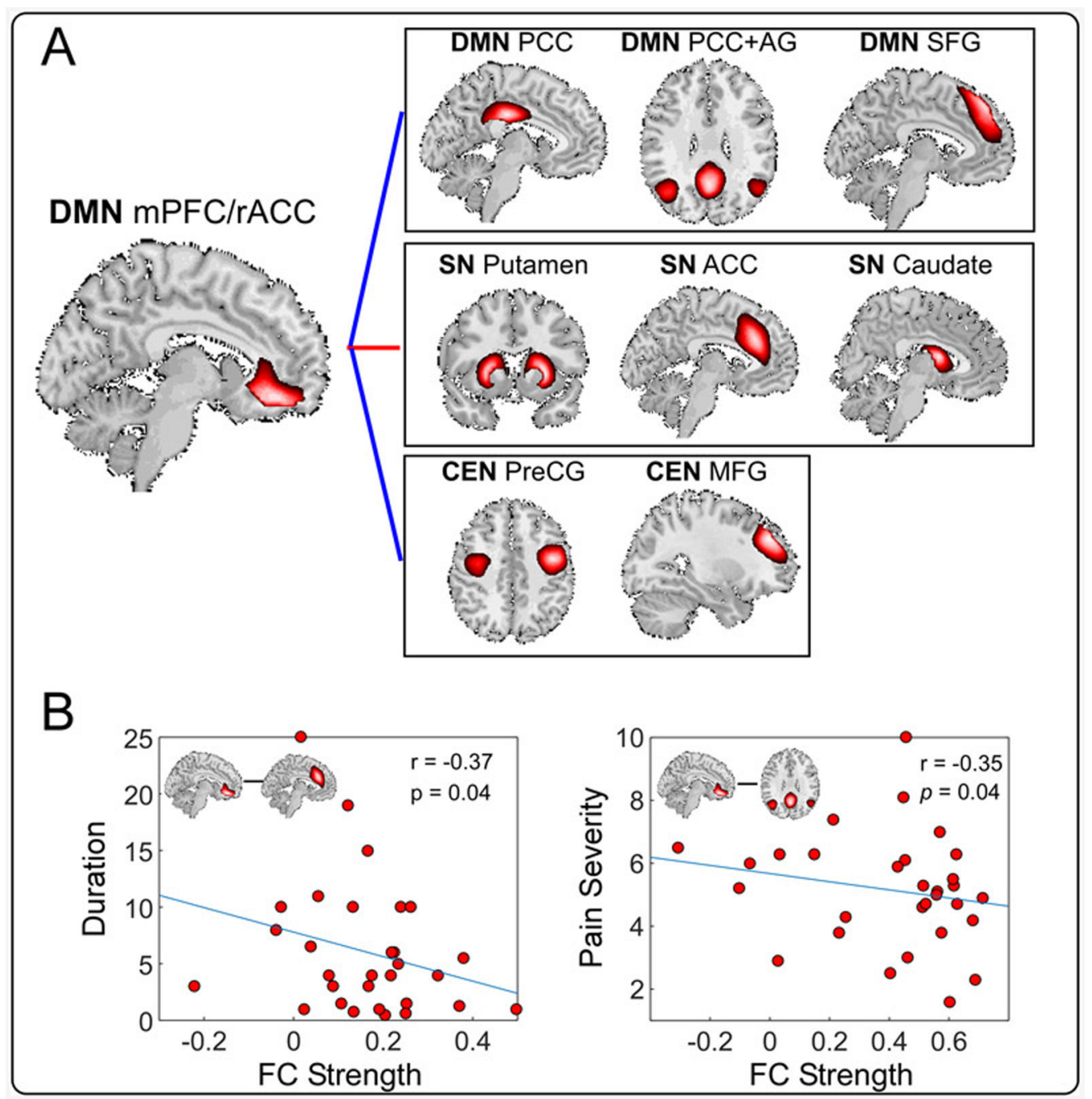

Figure 5.

Abnormal mPFC/rACC rsFC in cLBP from the validation data. Panel A: We found decreased $\mathrm{mPFC} / \mathrm{rACC}-\mathrm{DMN}$ and $\mathrm{mPFC} / \mathrm{rACC}-\mathrm{CEN}$ connectivity and increased $\mathrm{mPFC} /$ rACC-SN connectivity in cLBP patients. Panel B: mPFC/rACC-ACC rsFC was correlated with the duration of $\mathrm{cLBP}$, and $\mathrm{mPFC} / \mathrm{rACC}-\mathrm{PCC} / \mathrm{AG}$ rsFC was correlated with the severity of cLBP. SFG: superior frontal gyrus. 
A
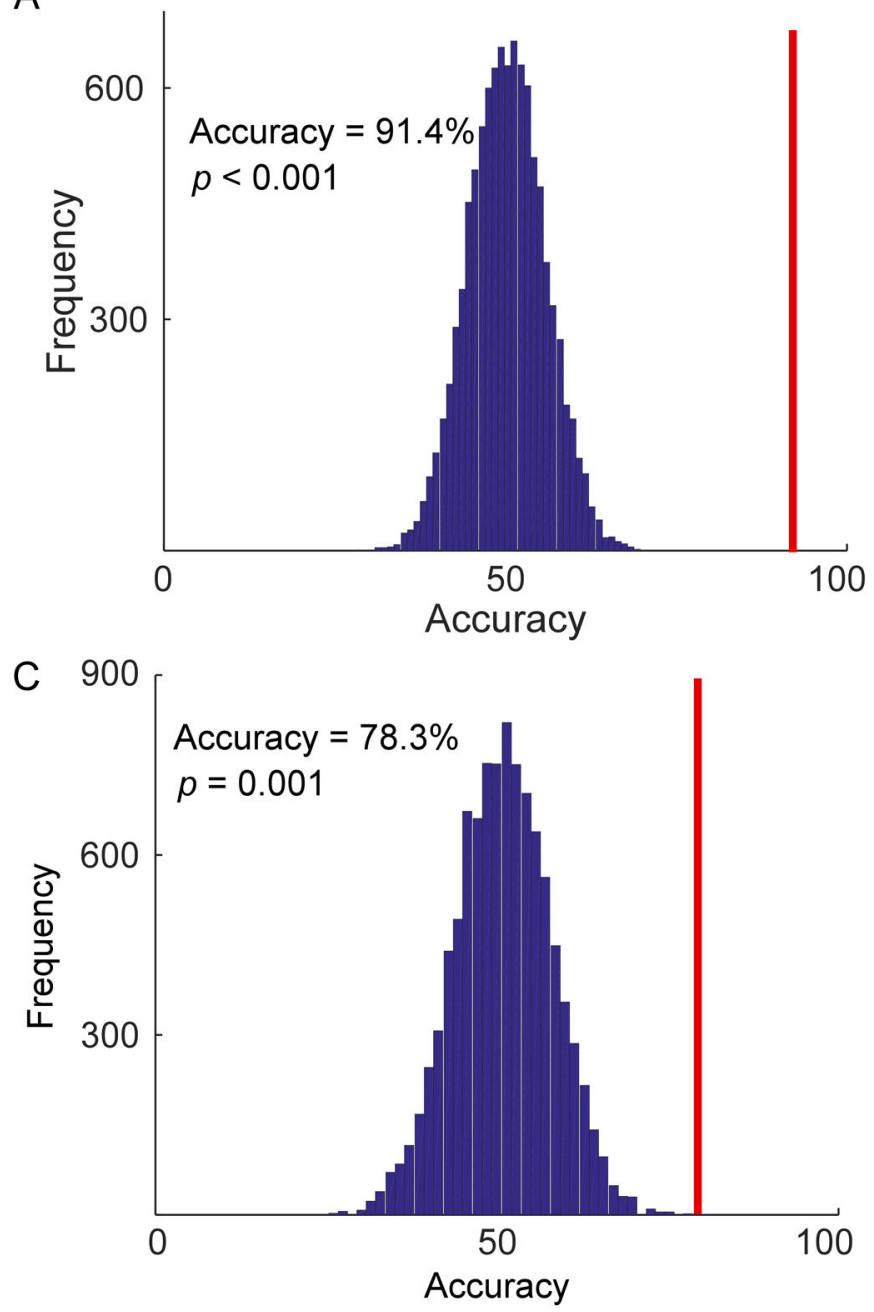

B
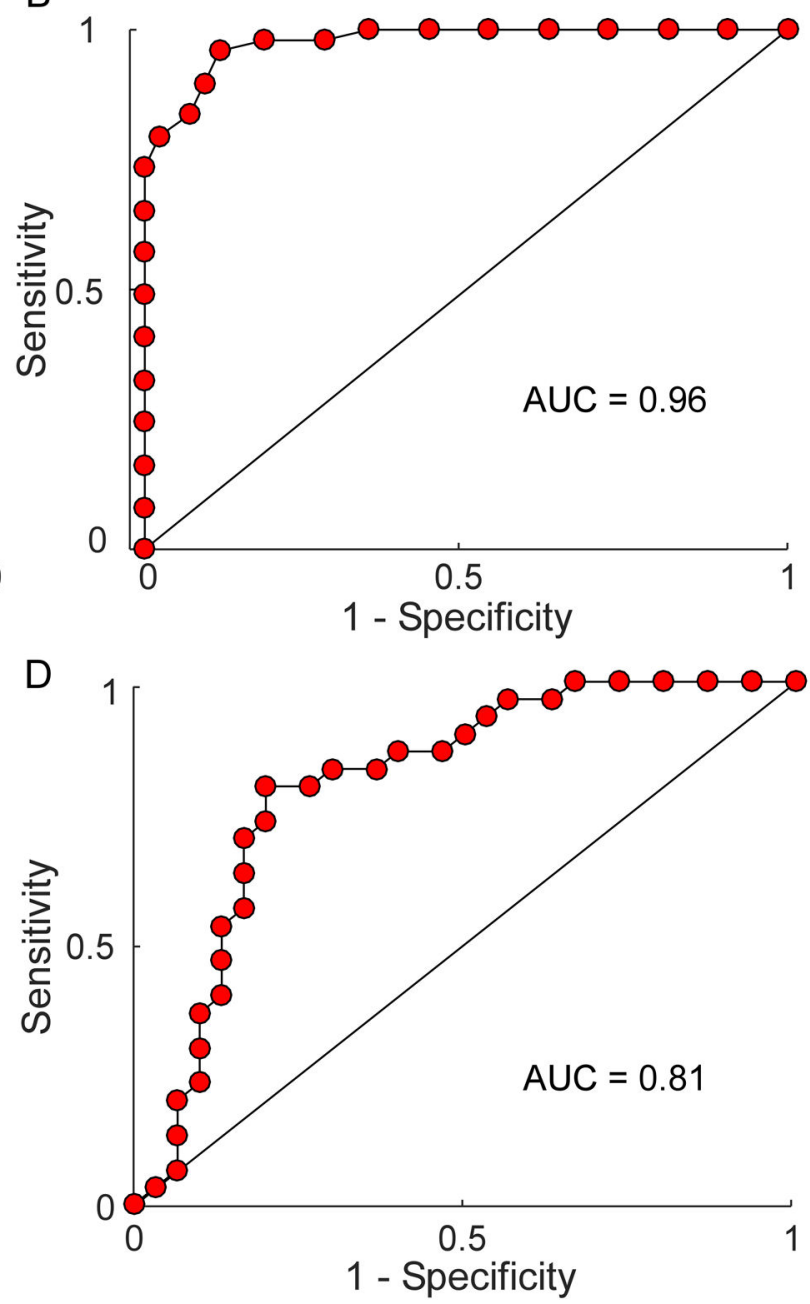

Figure 6.

Classification performance using rsFC. Panels A and B show that 5-fold cross-validation achieved an accuracy of $91.4 \%$ with $p<0.001$ and an AUC of 0.96 for discriminating cLBP patients from HCs. Panels $\mathrm{C}$ and $\mathrm{D}$ show that, in the independent dataset, the classifier achieved an accuracy of 78.3\% with $p=0.001$ and an AUC of 0.81 for discriminating cLBP patients from HCs. 
Table 1.

Demographics and clinical characteristics of cLBP patients and HCs in two datasets.

\begin{tabular}{lcccc}
\hline & cLBP (Dataset 1) & HC (Dataset 1) & cLBP (Dataset 2) & HC (Dataset 2) \\
\hline Demographics & 50 & 44 & 30 & 30 \\
No. subjects & $39.5 \pm 13.0$ & $36.9 \pm 8.2$ & $35.0 \pm 9.0$ & $34.2 \pm 1.5$ \\
Age & 31 females $(60 \%)$ & 19 females (43\%) & 14 females (47\%) & 16 females (53\%) \\
Gender & $8.0 \pm 7.4$ & $1.0 \pm 1.8$ & $4.1 \pm 4.0$ & $1.1 \pm 1.4$ \\
BDI & & & & \\
\hline Clinical symptoms & $5.6 \pm 1.7$ & & $5.3 \pm 1.6$ & \\
Pain severity score & $44.5 \pm 19.7$ & & $32.6 \pm 21.8$ & \\
Pain intensity & $61.3 \pm 5.6$ & & $59.2 \pm 5.4$ & \\
PROMIS Pain interference & $33.2 \pm 4.3$ & & $32.2 \pm 3.9$ & \\
PROMIS Physical function & $46.1 \pm 7.1$ & & $46.4 \pm 8.6$ & \\
PROMIS Social disability & $51.6 \pm 3.4$ & & $51.0 \pm 3.0$ & \\
PROMIS Sleep disturbance & $53.0 \pm 8.7$ & & $53.5 \pm 9.4$ & \\
PROMIS Fatigue & $51.4 \pm 7.9$ & $51.3 \pm 8.7$ & \\
PROMIS Anxiety & $48.2 \pm 8.1$ & $45.6 \pm 6.4$ & \\
PROMIS Depression & & &
\end{tabular}

*LBP: chronic low back pain; HC: healthy control; BDI: Beck depression inventory 